\title{
Integración de las TICs a la Cultura Docente
}

\author{
Integration OF the ICTS to the Teaching Culture
}

\author{
Rogelio Vilches Márquez \\ Especialista en Informática Educativa \\ Universidad de Chile \\ Capitán Ignacio Carrera Pinto 1045, Santiago \\ E-mail: rvilches@uchile.cl
}

\begin{abstract}
Resumen: Se propone abordar la integración curricular de las TICs a la cultura docente a partir de la transferencia de algunas de las metáforas de la Inteligencia Artificial y Vida Artificial. Los docentes experimentarían las TICs como el espacio natural para dominar algunos de los factores que determinan la complejidad de la organización escolar. Ello instalaría a la cultura docente en el centro de los paradigmas que dinamizan el actual desarmollo científico tecnológico.
\end{abstract}

Palabras clave: informática educativa, tecnologías de la información y comunicación (TICs), integración de tecnologías al currículo.

Abstract: The purpose of this paper is to approach the curricular integration of the ICTs to the teaching culture by means of transferring some metaphors of the Artificial Intelligence and Artificial Life. The teachers would experience the ICTs as the natural space to master some of the factors that determine the complexity of the school organization. This would install the teaching culture in the center of the paradigms that stimulate the present technological scientific development.

Keywords: informatics and education, information and communication technologies (ICTs), integration of technology into curriculum.

\section{INTRODUCCIÓN}

La literatura especializada documenta la insuficiente integración curricular de las tecnologías de información y comunicación (TICs) en las escuelas y liceos de nuestro país. Los usos que se hace de ellas, en muchos casos, terminan centrándose en la tecnología misma más que en las necesidades de aprendizaje de las organizaciones educacionales. Por ello, mucha de la investigación aún debe abocarse a definir el marco conceptual teórico de la integración de las TICs, más que a postular modelos de integración a partir de experiencias exitosas (Sánchez, 2003).

No poca de la integración curricular desarrollada hasta ahora se ha realizado desde la perspectiva de la transmisión de contenidos del currículo en formatos motivadores e interactivos. Sin embargo, las experiencias desarrolladas muestran que dichos usos de las TICs no superan la visión 
balcanizada de las asignaturas, propia de la cultura docente. Así, la Informática Educativa aparece más ligada a sectores de aprendizaje como matemática y ciencias en detrimento de los sectores 'humanistas' (DESUC, 2004).

Consideramos que los fenómenos antes descritos se correlacionan con un uso de las TICs centrado en la transmisión de información no generada en la propia organización educacional. Dicha realidad facilita el desarrollo de una relación pasiva entre Profesores y Tecnología. Al limitarse a evaluar la pertinencia de los contenidos transmitidos, los docentes se vuelven pasivos consumidores de productos desarrollados por expertos ajenos a la práctica cotidiana del aula. Ello envuelve un cierto contrasentido pues, si el objetivo de la escuela es preparar para vivir en la economía del conocimiento, resulta primordial que la organización educacional sea un espacio de construcción de conocimientos.

Hacer avanzar la relación de los profesores con las tecnologías de información y comunicación, desde un rol de consumidor de información a un rol de productor de conocimiento, supone que los profesores desarrollen una relación de dominio sobre las TICs. Un camino es introducir, dentro del perfil de las competencias docentes, las relacionadas con la programación de computadores. Dichas competencias están escasamente representadas en la cultura escolar de nuestro país, lo cual constituye una diferencia significativa respecto de los países que presentan altos puntajes en las mediciones internacionales sobre efectividad escolar (MINEDUC, 2002).

El desarrollo de competencias en programación de computadores podría renovar la cultura docente de nuestro país. Al desarrollar los profesores una relación de dominio creciente sobre las TICs, se apropiarían del núcleo de teorías centrales para el desarrollo científico-tecnológico actual. Pensamos en el paradigma evolucionista (Fischer, 2001).

\section{PROGRAMAR: ESO SUENA DIFÍCIL}

Sin embargo, ¿es realista plantear que los docentes pueden desarrollar habilidades en programación? ¿Acaso no deberían preocuparse de tareas mucho más prioritarias y pertinentes como mejorar los resultados de la escuela chilena en sectores estratégicos como lenguaje, matemática y ciencias?(OCDEMINEDUC, 2004). ¿Acaso no deberían mejorar sus capacidades de gestionar y administrar las instituciones educacionales? ¿Por qué priorizar algo que, a lo más, es sólo un recurso auxiliar para las tareas antes mencionadas y que no compensaría los costos de aprendizaje asociados?

Ciertamente, suena más razonable que los profesores dediquen su escaso tiempo disponible al aprendizaje de la ciencia que no manejan. Así, podrían mejorar sustancialmente los aprendizajes de los niños y niñas de nuestras escuelas. Parece un gran derroche de recursos dedicarse a aprender abstrusos 
lenguajes de programación más pertinentes a la formación de un ingeniero. Sin embargo, nos asalta la duda de si es posible aprender ciencia hoy sin aprender a manejar y programar simulaciones computacionales. El dominio de modelos computacionales es el camino que permite desarrollar experiencias de aprendizaje concreto respecto de teorías científicas ultra formalizadas (Resnick, 2001).

Si nos conformamos con comunidades de profesores que repiten lo que escucharon decir a un grupo de expertos sobre los resultados de las ciencias (en capacitaciones siempre apuradas y fuera de su entorno natural), no requerimos de profesores que dominen las TICs. Sin embargo, profesorestransmisores no parecen ser los modelos adecuados para que nuestros niños y adolescentes se inicien en el cultivo de las ciencias y comiencen su habitar en la sociedad del conocimiento.

Sin embargo, ¿es realista plantear el desarrollo de una cultura docente que domine las TICs a partir del desarrollo de competencias en programación, si los profesores no disponen de tiempo para aprender y, en general, no tienen acceso a computadores ni a redes adecuadas porque los sostenedores de escuelas y liceos no están dispuestos a solventar los costos fijos asociados? Puntualicemos que ninguna organización laboral ha dispuesto del tiempo ni de los recursos adecuados para incorporar las TICs a sus prácticas cotidianas, sin embargo, lo han hecho porque para sus integrantes había una ventaja estratégica asociada a esos aprendizajes. No cabría esperar algo muy diferente en la organización escolar. Los costos asociados al aprendizaje significativo de las TICs sólo serán asumidos por los educadores si las TICs agregan valor estratégico a su interacción con los distintos actores de la organización educacional. No parece ser que la actual integración curricular de las TICs, con su énfasis en la transmisión de contenidos (aún bajo ropajes constructivistas), mejore la situación estratégica de las comunidades de docentes.

Sin embargo, es realista plantear el desarmollo de una cultura docente que domine las TICs a partir del desarrollo de competencias en programación, si las herramientas informáticas están orientadas a mentes ingenieriles? ¿No estaríamos profundizando aún más la 'brecha digital' entre los sectores de aprendizaje 'científicos' y los 'humanistas' al desarrollar experiencias condenadas al fracaso?

Notemos que los profesores disponen de herramientas muy conocidas gracias a las capacitaciones desarrolladas por Enlaces: planillas de cálculo y bases de datos. Dichas herramientas de productividad tienen asociado un lenguaje de programación. Gracias a la familiaridad con dichos programas, no pocos profesores han desarrollando competencias en la programación de 'macros', tal como lo atestigua el sitio web de Educar Chile. 
Notemos, además, que existen lenguajes de programación producto de desarrollos en Inteligencia Artificial orientados a la cultura escolar. Por ejemplo, versiones del conocido lenguaje Logo orientado a la programación simbólica (Harvey, 1997a).

Al día de hoy, dichas herramientas han sido usadas o bien para el control administrativo o bien para reforzar los contenidos de los programas de matemática y tecnología. Proponemos explorar un enfoque alternativo: utilizar herramientas como las arriba mencionadas -y que ya están en nuestras escuelas- para gestionar el conocimiento pedagógico desarrollado por las comunidades de profesores. Más en concreto, utilizar dichas herramientas para simular en los computadores el funcionamiento de los modelos mentales propios de la cultura docente. Dichos modelos constituyen lo que en la literatura especializada se conoce como 'conocimiento tácito' (Nonaka y Takeuchi, 1999).

\section{MODELANDO LOS SABERES PEDAGÓGICOS}

Consideremos la realidad de muchos establecimientos de Enseñanza Media, cuya dinámica organizacional se desarrolla en torno a procesos de disciplinamiento y resistencia (Llaña y Escudero, 2003).

En dichos establecimientos, el logro significativo de aprendizajes ha dejado de ser el motor de la organización educacional. Los motivos son diversos. Sólo queremos mencionar uno: la percepción desarrollada por los profesores respecto de que sus alumnos carecen del capital cultural necesario para el logro de los objetivos demandados por el currículo.

La relación profesores-alumnado se ha cristalizado en un modelo mental, dominante en la cultura docente, que desarrolla en el profesor la 'realista' perspectiva de que sus esfuerzos por facilitar aprendizajes en sus alumnos fracasarán. Obviamente, la profecía tiende a autocumplirse.

¿Cómo manejar estos modelos entendidos como campos semánticos, que significan y re-significan las prácticas de la cultura docente? (Eco, 2000; Molina, 2004).

Proponemos desarrollar la experiencia de que los mismos docentes transformen sus modelos mentales en programas de computador, de manera que puedan percibirlos como objetos en funcionamiento, como artefactos que pueden ser modificados. Postulamos que experiencias de esa índole facilitarían tomar distancia de dichos modelos y someterlos a mutaciones.

Con un dialecto de LISP orientado a la computación simbólica, los profesores diseñarían artefactos (programas) capaces de 'dialogar' y 'razonar' 
(Harvey, 1997a y 1997b). Simularían, en el discurso, las relaciones de autoridad entre profesores y alumnos (Llaña y Escudero, 2003). Dichos programas simularían el 'discurso docente' y el 'discurso juvenil'. De esa manera, los educadores verían en funcionamiento los guiones que han generado para administrar la relación con sus alumnos.

En un segundo momento, los docentes introducirían 'mutaciones' en sus programas de simulación. Ello posibilitaría la experiencia, en el lenguaje, de mundos posibles alternativos. Por ejemplo, discursos no autoritarios para relacionarse con los alumnos. Los profesores construirían esta experiencia virtual de un modo seguro, serían los "patrones del buque" (Llaña y Escudero, 2003) y tendrían control sobre las variables asociadas a la conducta de sus alumnos. Este tipo de relaciones gratificantes relacionadas con las TICs, como experiencia de control sobre entornos complejos, está documentado en la investigación cualitativa (Turkle, 1984 y 1997). No sabemos que se haya considerado en la infoalfabetización de los pedagogos.

Supongamos que el programa de conversación le pidiera al profesor que caracterizara a sus alumnos. Esta petición podría formularse un número random de veces. Las respuestas que dé el profesor, en rigor marcas semánticas atribuidas al semema <<alumno > (Ec0, 2000), se guardarían en una variable como una lista de atributos. Luego el programa revisaría si entre los atributos figura la expresión <<privado de capital cultural $\gg$. Si la encuentra, pide que el profesor explique su sentido. Si entre las nuevas marcas semánticas encuentra algo como <<incapacidad de aprender >> el programa podría iniciar un diálogo que mostrara algunas de las competencias de los alumnos (manejo de TICs, habilidades físicas, formas de pensamiento estratégico desarrolladas en la cultura del videojuego, etc.). Es decir, el docente entraría en un discurso que desconstruye la caracterización de <<alumno >> como $<$ deprivado culturalmente $\gg$.

Estimamos que, experiencias como las esbozadas arriba, constituyen posibilidades de que la cultura docente re-descubra a las TICs como una oportunidad de re-significación de la relación alumno-profesor.

Ciertamente, procesos similares están abiertos para modelar la relación docente-apoderado, docente-directivo y docente-sostenedor. En este último caso, los lenguajes tipo LISP pueden ser complementados con programación de múltiples agentes, lo cual no deja de ser importante desde la perspectiva de una epistemología de la organización educacional, pues dichos lenguajes permiten una aproximación intuitiva al Dilema del Prisionero (Axelrod, 2003). Pensar la relación sostenedor-profesor desde la teoría de juegos remozanía la cultura docente. Una vez más encontramos herramientas computacionales de este tipo, orientadas a la cultura escolar (Wilensky, 2001). 


\section{CULTURA DOCENTE Y FORMACIÓN EN CIENCIAS}

Hacemos notar que el desarrollo de prácticas como las arriba mencionadas no apunta a la capacitación de los profesores de aula en ningún contenido científico para ser transferido a los alumnos con el fin de, supuestamente, preparar a dichos jóvenes para insertarse en la sociedad del conocimiento. Sin embargo, la experiencia de simular aspectos básicos de las interacciones estratégicas entre sostenedores y profesores (un proceso de negociación salarial, por ejemplo) sitúa en el centro de las prácticas docentes a las prácticas científicas. A partir de la resolución de los problemas de su cotidianidad profesional, los pedagogos se conectarían con categorías básicas de la teoría de juegos y de la complejidad, las cuales dinamizan el desarrollo científico tanto de las ciencias físico-matemáticas como de las ciencias sociales (Axelrod, 1986; Fischer, 2001).

El descubrimiento, por parte de los docentes, de que los computadores permiten desarrollar una nueva comprensión de las estrategias de los diferentes actores de la organización escolar y del propio sistema escolar, sería un aliciente para asumir el costo de aprender tecnologías inteligentes y el dedicar tiempo para un aprendizaje que probablemente no generará un ingreso directo para el profesor.

La comunidad de profesores de un Liceo, además, estaría en condiciones de transmitir la experiencia de sistematizar sus propios saberes, esta vez a sus propios alumnos. En ese caso el rol docente se asimilaría al de un asesor que pone su experticia al servicio de la resolución de los conflictos propios de la cotidianidad juvenil. Ahora los alumnos descubrinán la potencialidad de modelar fenómenos como las identidades grupales, el consumo de drogas, la ecología del carrete, etc.

Alumnos y profesores experimentarían y usarían algunas de las analogías epistemológicas más potentes de la hora presente. La problemática cotidiana de la escuela aparecería naturalmente conectada con la mecánica cuántica, la biología evolucionista, la dinámica de las fuerzas del mercado y el funcionamiento de las empresas (Fischer, 2001).

\section{UN RECURSO PARA LA FORMACIÓN INICIAL DOCENTE}

Introducir la programación apoyándose en metáforas desarrolladas por la Inteligencia Artificial permite explorar un camino complementario para resolver una de las piedras de toque de la formación inicial docente: el problema de superar el paradigma del profesor como transmisor de contenidos (Inostroza, 1996). 
En el marco de la renovación del currículo para la formación inicial del pedagogo, se han emprendido serias intervenciones para modificar los modelos mentales con los cuales los alumnos de Pedagogía significan sus prácticas docentes (Inostroza, 1996; Suzuki et al., 2004). A través de talleres de reflexión, en muchos casos bajo la guía de la investigación-acción, los profesores formadores de profesores han procurado gatillar procesos metacognitivos que permitan a los futuros educadores tomar conciencia de los modelos mentales inadecuados con los que tienden a enfrentar sus prácticas al interior de la cultura escolar. Las metodologías ocupadas se inscriben en el uso del diálogo y la escritura reflexiva. Tras unos años de desarrollo de estas expeniencias, algunos investigadores comienzan a manifestar su insatisfacción respecto de los resultados obtenidos (Molina, 2004).

Postulamos la siguiente explicación del fenómeno: el diálogo crítico y la escritura reflexiva no provocan el suficiente distanciamiento del alumno de pedagogía de sus modelos mentales. Si bien puede hacer una primera toma de conciencia, en rigor, no tiene posibilidad de probar variaciones (mutaciones) en sus modelos de significación. En ese sentido, la escritura y el papel muestran sus características más orientadas a la fijación y representación estática de estructuras de significación. Ni la escritura ni el papel facilitan la interacción y manipulación de dichos modelos.

Simular dichas estructuras con un lenguaje de programación adecuado resuelve las limitaciones antes señaladas. Un modelo mental transformado en software adquiere la propiedad de que puede correrse (ejecutarse). Se lo puede ver en funcionamiento 'fuera de la mente', en un dispositivo físico y mecánico. Este proceso de objetivación permite que el docente juegue con sus contenidos mentales, juegue a modificarlos y explore el mundo posible que aparece.

Las prácticas crítico-reflexivas, necesarias para la formación de profesores en la economía del conocimiento, encuentran en las TICs la tecnología adecuada para sistematizar la experiencia profesional y producir el saber pedagógico. De este modo, las tecnologías (bajo la permanente sospecha de ser herramientas al servicio del control experto 0 administrativo) apoyanían la formación de educadores capaces de desarrollar autonomía en la búsqueda de las soluciones a los desafíos de la cultura escolar (Editor, 2004).

\section{CONCLUSIÓN: PERSPECTIVAS PARA LA INVESTIGACIÓN EDUCACIONAL}

Los simuladores desarrollados por los docentes integrados en la organización educacional y por los alumnos de pedagogía, abrirían todo un 
campo para la investigación educacional. Permitiría a los investigadores abordar, bajo una nueva perspectiva, preguntas de investigación como:

- ¿Cuáles son los presupuestos típicos que un profesor activa en la interacción con sus alumnos?

- ¿Qué menú de respuestas tiene un profesor para los requerimientos que enfrenta en la escuela?

- ¿Qué características tendría la interacción del docente con un programa de computador que simula sus estrategias-alumno?

- Si el computador asume el 'rol-estratégico-docente' y el docente el 'rol-(virtual)- alumno', ise modificará su comprensión de la cultura juvenil?

- ¿Presentará el modelo computacional 'interacción alumno-profesor' isomorfismos con el modelo 'interacción profesor-directivo' y el modelo 'interacción profesor-sostenedor'?

Muchas de estas interrogantes se abordan, habitualmente, desde una perspectiva cualitativa. El modelamiento computacional de los hallazgos de la investigación cualitativa permitiría tender un puente con la investigación cuantitativa.

Por ejemplo, el campo semántico 'interacción con el alumno' (operativo en la cultura docente), se puede construir desde la interpretación de registros etnográficos, focus groups, etc. Los investigadores crearían simulaciones computacionales que funcionen de acuerdo al modelo establecido cualitativamente. Enseguida se invitaría a profesores de aula a jugar con los simuladores. Ello permitiría probar su efectividad e irlos depurando. El resultado de esas interacciones se almacenaría en variables, con lo cual habría una recolección de datos susceptibles de tratamiento estadístico. Fácilmente se podrían calcular frecuencias respecto del uso de tales o cuales conceptos (en rigor, marcas semánticas). Más aún, el cálculo de correlaciones entre parejas de marcas semánticas permitináa reconstruir el espacio semántico de la cultura docente (Eco, 2000).

El periplo epistemológico que bosquejamos arriba, con el auxilio de la programación de múltiples agentes, permitiría, inicialmente, conectar la investigación educacional con nuevas propuestas desarrolladas para el campo de las ciencias sociales. Nos referimos a aquellas que proponen aislar las relaciones fundamentales que caracterizan los micromotivos de los individuos, desde los cuales emergen las macroconductas de las organizaciones humanas (Axelrod, 2003). 


\section{REFERENCIAS BIBLIOGRÁFICAS}

Axelrod, R., 1986

Axelrod, R., 2003

DESUC, 2004

Eco, U., 2000

Editor, 2004

Fischer, A., 2001

Harvey, B., 1997a

Harvey, B. 1997b

Inostroza, G. 1996

Llaña, M. y E. Escudero, 2003

MINEDUC, 2002

Molina, P., 2004

Nonaka, I. e H. Takeuchi, 1999

OCDE-MINEDUC, 2004

Resnick, M., 2001

Rittershaussen, S., I. Contreras, E. Suzuki, M. C. Solís \& P. Valverde, 2004

Sánchez, J., 2003

Turkle, S., 1984
La evolución dela cooperación. Alianza Editorial, Madrid. $211 \mathrm{pp}$.

La complejidad de la cooperación. FCE, México D.F. $279 \mathrm{pp}$.

Uso de recursos informáticos por parte de los docentes. PUC, Santiago. 9pp.

Tratado de semiótica general. Editorial Lumen, Barcelona. $461 \mathrm{pp}$.

Editorial. En: Revista Enfoques Educacionales, Santiago, 6 (1): 5-8.

Evolución... el nuevo paradigma. Editorial Universitaria, Santiago.171 pp.

Computer Science Logo Style. V1: Symbolic Computing. MIT Press. 318 pp.

Reasoning with Computers. Ver. http://www.cs.berkeley.edu/ -bh/logic.html

Talleres pedagógicos. Dolmen Ediciones, Santiago. $185 \mathrm{pp}$.

Alumnos y profesores: resonancia de un desencuentro. Departamento de Educación, Universidad de Chile, Santiago. $126 \mathrm{pp}$.

Informe STTES: el caso de Chile. MINEDUC, Santiago. $23 \mathrm{pp}$.

Talleres pedagógicos: un espacio para el desarrollo de la reflexión en la formación inicial. En: Revista Pensamiento Educativo, Santiago. 35: 161-172.

La organización creadora de conocimiento. Cómo las compañías japonesas crean la dinámica de la innovación. Oxford University Press, México D.F. $318 \mathrm{pp}$.

Balance de la reforma educacional Chilena. MINEDUC, Santiago. 400 pp.

Tortugas, termitasy atascos de tráfico. Gedisa, Barcelona. $222 \mathrm{pp}$.

Prácticas iniciales en la formación de educadores. En: Revista Pensamiento Educativo, Santiago, 35 ( $2^{\circ}$ semestre de 2004): 192-215.

Integración curricular de TICs. Concepto y modelos. En: Revista Enfoques Educacionales, Santiago. 5 (1): 51-65.

El segundo yo. Ediciones Galápago, Buenos Aires. $349 \mathrm{pp}$. 
Turkle, S., 1997

Wilensky, U., 2001
La vida en la pantalla. Ediciones Paidós, Barcelona. $414 \mathrm{pp}$.

Emergent Entities and Emergent Processes: Constructing Emergence through Multi-agent Programming. En Annual Conference of the American Educational Research Association, Seattle, WA. Ver en: http:// ccl.northwestern.edu/papers/AERA/ 